\title{
Predictive value of hepatic transaminases during febrile phase as a predictor of a severe form of Dengue: analysis of adult Dengue patients from a tertiary care setting of Sri Lanka
}

\author{
Dickowita Kankanamge Dilani Priyangika' , Gayani Premawansa², Madura Adikari², Sharmila Thillainathan², \\ Sunil Premawansa ${ }^{3}$, Bernard Deepal Wanniarachchi Jayamanne ${ }^{4^{*}}$ (D) and Ranjan Premaratna ${ }^{5}$
}

\begin{abstract}
Objectives: Dengue viral infection is an ongoing epidemic in Sri Lanka, causing significant mortality and morbidity. A descriptive-analytical study was carried out using serologically confirmed Dengue patients during a 6 month period. The relationship between the elevation of hepatic enzymes and severity of Dengue was assessed after stratifying recorded maximum AST/ALT (SGOT/SGPT) values 2-15 times elevated and by the phases of the illness. Sensitivity, specificity, predictive values, and ROC curves were assessed using maximum values for AST and ALT.

Results: Out of 255 patients, 107(42\%) were females. The majority (52.9\%) were in the 20-39 year age group. Only $19.6 \%$ had DHF. No statistically significant difference was noticed in the values of maximum transaminases during the febrile phase among DF and DHF patients. Higher sensitivity and low specificity with the 1-5 times elevation range was noticed, and a higher cut-off level of more than 5 times elevation showed low sensitivity and higher specificity. The combination of both transaminases cut-offs with age and sex also does not show clinically significant predictability of severe disease.

The AST and ALT elevations are not showing discriminatory predictive value on dengue severity. As different serotypes cause different epidemics, it is important to carry out large-scale specific studies considering the serotypes.
\end{abstract}

Keywords: Transaminases in Dengue, Dengue severity prediction, Dengue, And liver enzymes

\section{Introduction}

Dengue Virus infection is known to cause significant morbidity and mortality in Sri Lanka. In 2017, total of 186,101 dengue cases were reported [1] with 440 deaths $[1,2]$. It has been well reported in all districts of Sri Lanka and contributes to a substantial proportion of health costs [3]. The clinical entity of the disease can range from

\footnotetext{
${ }^{*}$ Correspondence: bdw.jayamanne@kln.ac.lk

${ }^{4}$ Department of Public Health, Faculty of Medicine, University of Kelaniya, Kelaniya, Sri Lanka

Full list of author information is available at the end of the article
}

simple dengue fever, dengue haemorrhagic fever, and dengue shock syndrome.

Liver involvement in dengue infection is well described; however, evidence is limited whether the elevation of liver transaminases (AST-Aspartate transaminase or SGOT-Serum glutamic oxaloacetic transaminase and ALT-Alanine transaminase or SGPT-Serum glutamate-pyruvate transaminase) can be related to the severity of the disease. In 2009, the World Health Organization (WHO) revised its dengue guidelines and proposed severe organ impairment as one category of severe 
dengue in addition to severe plasma leakage and severe bleeding [4].

While some researchers show that elevated liver enzymes are a feature of severity, some other researchers concluded that it could occur at any point of the disease. Since there were limited studies carried out on this issue, the necessity of conducting a proper study has arisen to assess the relationship between elevated liver enzymes and the severity of the disease $[5,6]$.

If a relationship between elevations of liver transaminases and disease severity is elicited, liver transaminase elevation can be used as a predictor of disease severity, and those patients can be kept under close observation. Predicting the disease severity and prior preparedness and close monitoring will reduce future disease-related mortality.

\section{Ethical considerations and approval}

Ethical approval was obtained by the Ethical Review Committee Faculty of Medicine, University of Kelaniya, Sri Lanka(P/75/04/2014). All participants gave informed written consent. Identifying information was kept entirely confidential.

\section{Study design and setting}

A descriptive-analytical study was carried out at Colombo North Teaching Hospital (CNTH), Ragama, Sri Lanka. Data were collected for six months from 01st of May to 30th of October 2014.

\section{Inclusion criteria}

All the patients who were confirmed using NS1dengue antigen and admitted to the two medical units were included in the study after obtaining informed written consent.

\section{Exclusion criteria}

Patients with acute and chronic liver diseases were excluded from the study.

\section{Confirmation of diagnosis}

Dengue was confirmed by clinical case definition along with Dengue NS1 antigen detection on acute serum samples. After considering serial clinical and laboratory data from the entire clinical course of the patient, treating clinicians categorised patients as having dengue fever (DF), Dengue haemorrhagic fever (DHF), or dengue shock syndrome (DSS) using WHO classifications 4, 6]. Dengue haemorrhagic fever required evidence of plasma leakage with or without other abnormal haematological parameters in addition to DF [4]. Dengue shock syndrome was defined when DHF was associated with either tachycardia or pulse pressure $<20 \mathrm{mmHg}$ or systolic blood pressure $<90 \mathrm{mmHg}$ [7].

\section{Data collection}

Data were collected by using a questionnaire for patient interviews and a data extraction sheet for medical records.

\section{Data analysis}

According to the laboratory standards, a level of $35 \mathrm{IU} / \mathrm{l}$ was taken as the upper limit of normal (ULN) for both AST and ALT. Subgroup analysis was carried out febrile phase subset using maximum AST and ALT (SGOT and SGPT) values after stratifying for three phases of dengue; febrile (days 1-3 of illness), critical (days 4-6), and convalescent (days 7-14) phases as defined by WHO 2009 [4]. Maximum AST and ALT values that occurred during the febrile phase form 2-15-fold rise (with ULN) were considered for analysis.

Statistical significance for continuous variables was determined using the Mann-Whitney $\mathrm{U}$ test. $\mathrm{P}$ value of 0.05 was taken as the significant cut off value. To figure out discriminatory performance, Receiver Operating Characteristic (ROC) curves were used in addition to screening statistics (Sensitivity, Specificity, Positive Predictive Value (PPV) and Negative Predictive Value (NPV).

Binary logistic regression also performed to assess the combined ability of two transaminases and combination with age and sex for the patients who showed either of maximum transaminases elevation for 3 categories (Normal, between 5 and tenfold rise and greater than tenfold rise) $[8,9]$ during the febrile phase of the disease. Data was analysed by SPSS (Version 22).

\section{Results}

A total of 255 patients were recruited for the study. The majority were males (58\%). Median age 27 years $(\mathrm{Q} 1=21, \mathrm{Q} 3=40, \mathrm{IQR}=19)$. Dengue haemorrhagic fever was detected in fifty patients (19.6\%). There were no cases of dengue shock syndrome. 14(5.5\%) of all patients had bleeding manifestations, out of which $50 \%$ were DHF patients.

In the uncomplicated dengue fever category, males in the age group 20- 29 years were the highest prevalent group (19.2\%) among the sample, while the least prevalent age-sex group was 60 years and above male group (1.6\%) (Table 1). In the DHF group, females of the age 30-39 years had the highest prevalence (3.9\%) while males of 60 years and above had the least prevalence (0.0\%) (Table 1).

Maximum ALT and AST values have occurred during the febrile phase among 59.2\% (141/255) and 62.5\% 
Table 1 Age categories of DF and DHF patients

\begin{tabular}{|c|c|c|c|c|c|c|}
\hline \multirow[t]{2}{*}{ Age category (years) } & \multicolumn{2}{|l|}{ Males } & \multicolumn{2}{|l|}{ Females } & \multicolumn{2}{|l|}{ Total } \\
\hline & DF (\%) & DHF (\%) & DF (\%) & DHF (\%) & DF (\%) & DHF (\%) \\
\hline $12-19$ & $31(12.6)$ & $04(1.6)$ & $11(04.3)$ & $09(3.5)$ & $42(16.5)$ & $13(5.1)$ \\
\hline $20-29$ & 49 (19.2) & $07(2.8)$ & $21(08.2)$ & $06(2.4)$ & $70(27.4)$ & $13(5.1)$ \\
\hline $30-39$ & $25(09.8)$ & $02(0.8)$ & $15(05.9)$ & $10(3.9)$ & $40(15.7)$ & $12(4.7)$ \\
\hline $40-49$ & $15(05.9)$ & $02(0.8)$ & 09 (03.5) & $02(0.8)$ & $24(09.4)$ & $04(1.6)$ \\
\hline $50-59$ & $08(03.1)$ & $01(0.4)$ & 09 (03.5) & $02(0.8)$ & $17(06.7)$ & $03(1.2)$ \\
\hline Above 59 & 04 (01.6) & $00(0.0)$ & $08(03.1)$ & $05(2.0)$ & $12(04.7)$ & $05(2.0)$ \\
\hline Total & $132(51.8)$ & $16(06.3)$ & $73(28.6)$ & $34(13.3)$ & $205(80.4)$ & $50(19.6)$ \\
\hline
\end{tabular}

$(159 / 255)$ patients, respectively (Additional file 1: Table S1). The subgroup of patients who showed a maximum rise of one or both transaminases during the febrile phase, comparing medians of both transaminases of DF and DHF patients, did not show a statistically significant difference. (Additional file 2: Table S2). Further analysis of the area under the curves (AUC) of ROCs for different cut-offs is shown in Fig. 1. All cut-off levels for both transaminases showed an area under the curve values within the range of $0.7-0.4$ with a descending trend towards the high cut-off values (Fig. 1).

Specificity vs sensitivity, plot shows AST lies at the specificity and accuracy triangle while ALT lies at the other half [10] (Fig. 2). All screening parameters showed the reach of their saturated value after an eightfold rise in both transaminases (Additional file 4: Figure S1 and Additional file 5: Figure S2).

Combined logistic regression models for the subgroup who had maximum elevation of transaminases during the febrile phase also showed statistically insignificant parameter estimates for both transaminases (AST $p$ value $=0.712$, ALT $p$ value 0.701 ) at continuous scale alone. Adding age and sex did not improve the model $($ AST $p$ value $=0.917$, ALT $p$ value 0.852). However, parameter estimate for sex had a significant predictive value for DHF in the model showing a significant Odds ratio $(\mathrm{p}$ value $=0.002)$ (Additional file 3: Table S3).

\section{Discussion}

In this study, the maximum elevation of transaminases seen during the febrile phase was around $60 \%$ of the sample. Cut-off levels for both transaminases showed an area under the curve values within the range of $0.7-0.4$ with a descending trend towards the high cut off values. Combined logistic regression models also showed statistically insignificant parameter estimates for both transaminases.

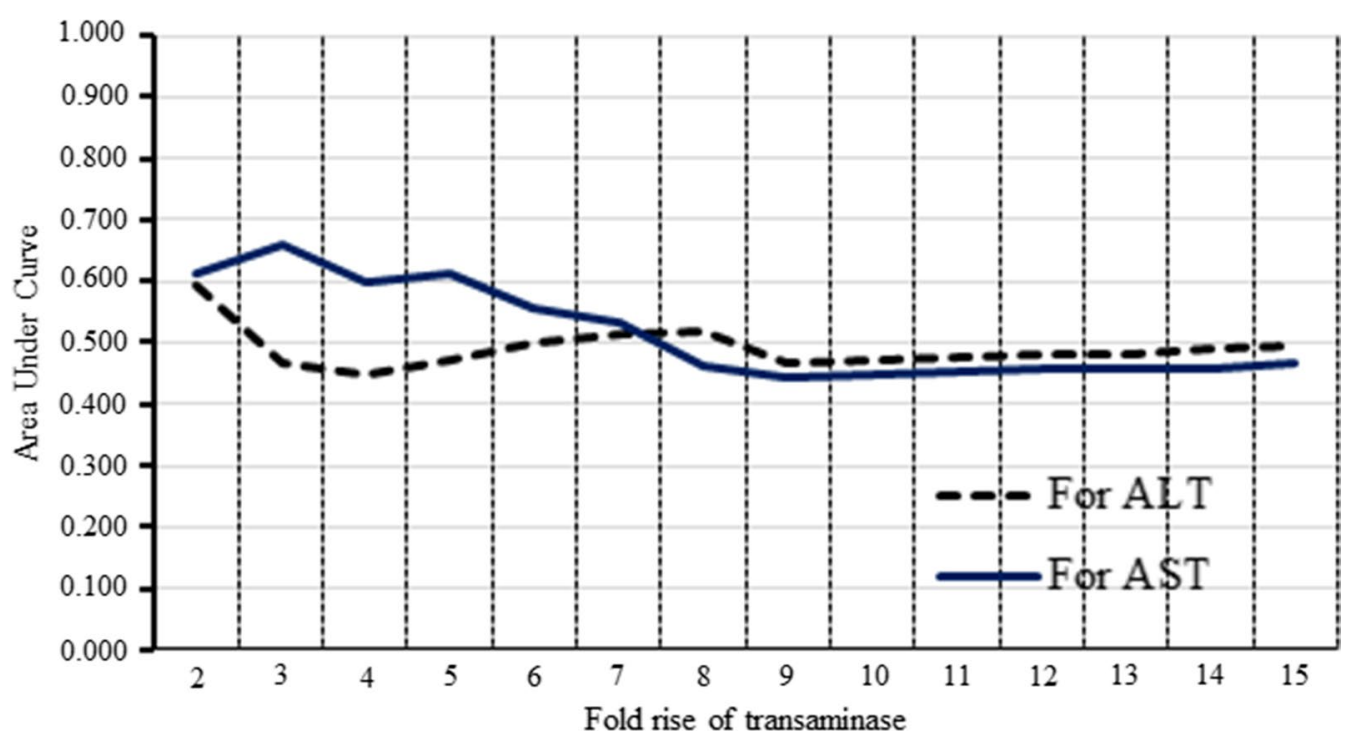

Fig. 1 Area under the curve against fold rise of AST and ALT 


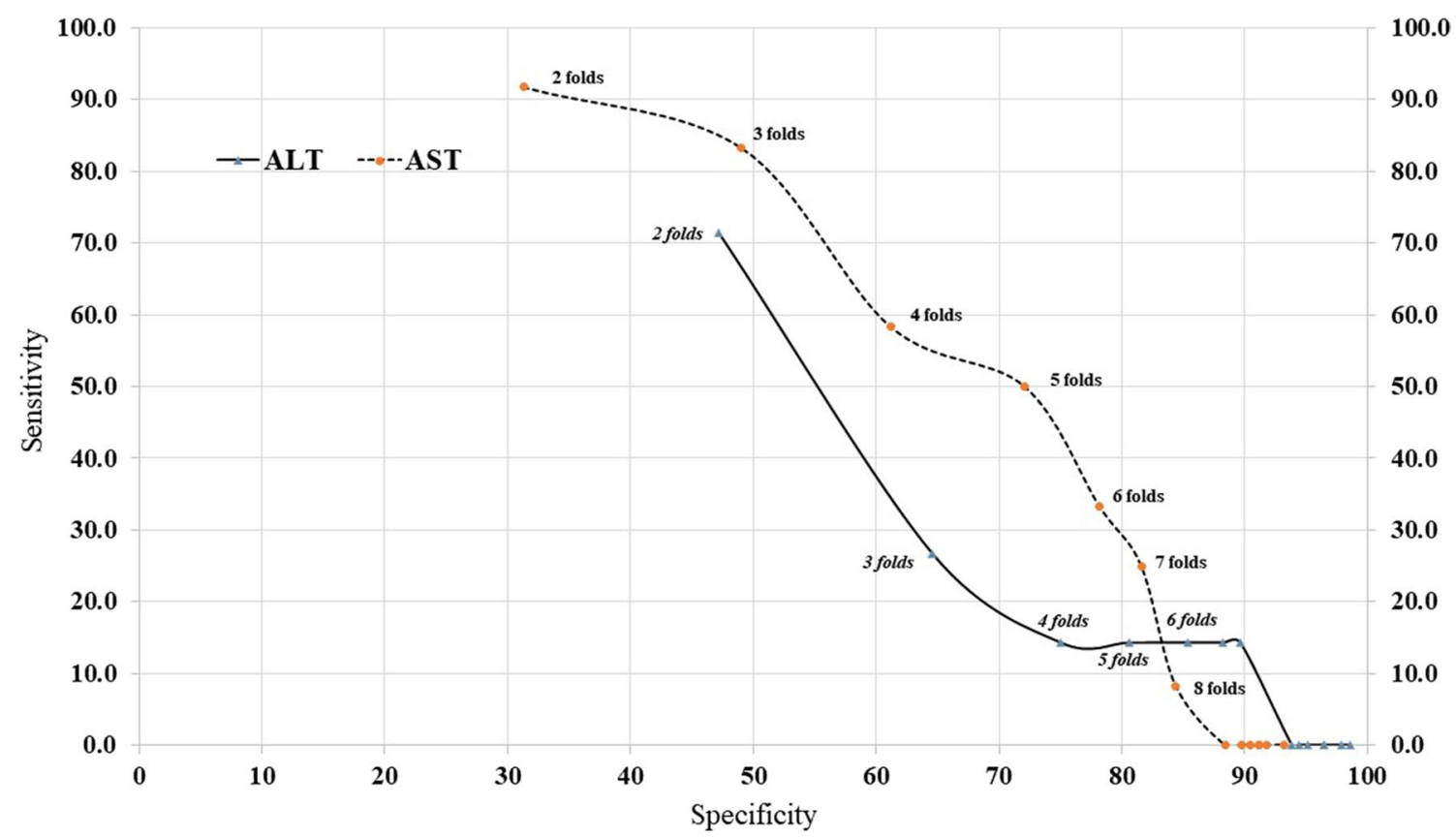

Fig. 2 Specificity against Sensitivity for both transaminases all cutoff levels

Acute liver is uncommon in dengue fever and was not reported in this study population. According to a study done by Trung and Kuo, only $1.1 \%$ had an acute liver failure with dengue infection $[7,11]$.

In the subgroup of patients who showed elevated transaminases during the febrile phase, a small proportion of DHF patients have shown the highest level during the febrile phase (Fig. 2). The median transaminases levels have shown no significant difference between DF and DHF patients (Additional file 3: Table S3). Liver function tests done at earlier dates might not reflect the extent of liver involvement in acute infection [12]. Analysis of statistics showed in Additional file 2: Table S2. So, there is no discriminatory value for median cut-off levels of transaminases.

According to ROC curve values, AUC between 0.9 and 1 considered as best predictors, $0.8-0.9$ good predictors, $0.7-0.8$ fair predictors, $<0.7$ not good tests. Analysis of ROC curves using our data (Fig. 1), showed an area under the curve values below 0.7, which shows all the cut-offs fall into the "not good test" category.

There are only a few studies done and limited data available to find whether elevation of liver enzymes can be used as a predictor for DHF, including this study (Additional file 4: Figure S1 and Additional file 5: Figure S2). It was noted that results are varied, and many of the studies have shown that AST and ALT values cannot predict severity, while some studies have shown it has a certain degree of predictability as an independent predictor of DHF [13]. Some studies have shown that AST and ALT values may differ between DF and DHF $[5-7,14]$, few studies support AST or ALT as an independent predictor of DHF [10].

The exact reasons for elevation in liver enzymes in dengue fever are unclear. ALT is more specific for liver cell injury. Varying degrees of liver involvement is seen during acute dengue infection and are thought to result from hepatocyte apoptosis directly by the virus, hypoxic damage due to impaired liver perfusion resulting from fluid leakage, oxidative stress, or immune mediated injury [12]. On the other hand, elevation in AST can occur due to multiple pathologies involving the heart, muscle, red blood cells, kidneys, brain, and liver $[15,16]$.

\section{Conclusions and recommendations}

In conclusion, the elevation of liver transaminases is common in dengue infection, however, use of elevated transaminases during the febrile phase alone is not a good predictor of the disease severity as a quick initial assessment. Combining it with one or more laboratory parameters like lowest total white cell count, lowest platelet count, etc. may be a good pathway for further evaluation and the timing of the febrile phase (day of fever). As different dengue virus serotypes cause different epidemics, it is also important to carry out further research in detail to explore the association of disease 
severity and elevated transaminases in that aspect as well with dengue patients.

This study was carried out among adults, and further studies in different age groups, including children, are suggested.

\section{Limitations}

Since it is needed to find severity before the occurrence of critical phase, maximum AST/ALT value during the total disease course (occurred mostly during critical phase) cannot be used for clinical decision-making and predicting disease progression. Due to limited investigation facilities available in the public sector set up, it was not possible to assess co-infection with hepatitis viruses and hepatotoxicity. Patients who are known to have liver pathologies were excluded from the study during the time of selection. Also, screening for other virus infections was not routinely carried out due to the limited availability of the facilities.

\section{Abbreviations}

ALT: Alanine transaminase; AST: Aspartate transaminase; AUC: Area under curve; CNTH: Colombo North Teaching Hospital; DF: Dengue fever; DHF: Dengue haemorrhagic fever; DSS: Dengue shock syndrome; NPV: Negative predictive value; PCR: Polymerase chain reaction; PPV: Positive predictive value; ROC: Respondent operative characteristics; SGOT: Serum glutamic oxaloacetic transaminase; SGPT: Serum glutamate-pyruvate transaminase; ULN: Upper limit of normal; WHO: World Health Organization.

\section{Supplementary Information}

The online version contains supplementary material available at https://doi. org/10.1186/s13104-021-05670-0.

Additional file 1: Table S1: Maximum transaminases levels in different phases

Additional file 2: Table S2: Median comparisons (by Mann-Whitney U test)of the febrile phase maximum transaminases levels

Additional file 3: Table S3: Parameter estimates for binary logistic regression model for DF/DHF prediction from both transaminases, age and sex variables

Additional file 4: Figure S1: Screening statistics with fold rise of ALT

Additional file 5: Figure S2: Screening statistics with fold rise of AST

\section{Acknowledgements}

Our acknowledgement goes to all the patients who participated the research. We would like to acknowledge Dr Madura Adikari and Dr Sharmila Pradeep for their extended valuable support throughout the research study. In addition, our acknowledgement is extended to microbiologist and their team and laboratory staff for the immense support in the investigations. Dr. C. Rodrigo of UNSW Sydney, 2052, Sydney, NSW, Australia.

This study was done in order to fulfillment of the requirement for Board Certification as a Consultant Physician at the Postgraduate Institute of Medicine, University of Colombo and has not published or submitted for publication elsewhere.

\section{Authors' contributions}

Conception or design of the work by DKDP, RP and GP. Data collection by DKDP, MA and ST. Data analysis and interpretation by BDWJ, DKDP, RP,GP and
SP. All the authors involved in drafting the article and critical revisions of the article. All authors contributed to the background, design, and drafting of the study proposal, conducting the study and preparing the manuscript. All authors read and approved the final manuscript.

\section{Funding}

Self funded by the authors.

\section{Availability of data and materials}

The datasets generated during and/or analysed during the current study are not publicly available, but are available from the corresponding author on reasonable request.

\section{Declarations}

\section{Ethical approval and consent to participate}

Ethical approval was obtained by the Ethical Review Committee, Faculty of Medicine, University of Kelaniya under P/75/04/2014, Sri Lanka. Prior informed written consent was obtained from the participants.

\section{Consent for publication}

Not applicable.

\section{Competing interest}

No financial and non-financial competing interests to declare.

\section{Author details}

${ }^{1}$ University Medical Unit, Teaching Hospital, Ragama, Sri Lanka. ${ }^{2}$ Colombo North Teaching Hospital, Ragama, Sri Lanka. ${ }^{3}$ Zoology and Environmental Science, Faculty of Science, University of Colombo, Colombo, Sri Lanka. ${ }^{4}$ Department of Public Health, Faculty of Medicine, University of Kelaniya, Kelaniya, Sri Lanka. ${ }^{5}$ Department of Medicine, Faculty of Medicine, University of Kelaniya, Kelaniya, Sri Lanka.

Received: 2 January 2021 Accepted: 23 June 2021

Published online: 30 June 2021

\section{References}

1. Epidemiology Unit, Sri Lanka. Epidemiology Unit-Disease Surveillance-Trends. Epidemiology Unit, Sri Lanka. http://www.epid.gov.Ik/ web/index.php?option=com_casesanddeaths\&ltemid=448\&lang=en.

2. Tissera HA, Jayamanne BDW, Raut $R$, Janaki SMD, Tozan $Y$, Samaraweera PC, et al. Severe Dengue epidemic, Sri Lanka, 2017. Emerg Infect Dis. 2020;26(4):682-91.

3. Thalagala N, Tissera H, Palihawadana P, Amarasinghe A, Ambagahawita A, Wilder-Smith A, et al. Costs of Dengue control activities and hospitalizations in the public health sector during an epidemic year in urban Sri Lanka. PLOS Negl Trop Dis. 2016;10(2): e0004466.

4. World Health Organization (WHO). WHO | Dengue guidelines for diagnosis, treatment, prevention and control: new edition. 2009. http://www. who.int/rpc/guidelines/9789241547871/en/. Accessed 25 Jul 2018.

5. Lee LK, Gan VC, Lee VJ, Tan AS, Leo YS, Lye DC. Clinical relevance and discriminatory value of elevated liver aminotransferase levels for dengue severity. PLOS Negl Trop Dis. 2012;6(6): e1676.

6. de Souza $\sqcup$, Alves JG, Nogueira RMR, Gicovate Neto C, Bastos DA, Siqueira EWdS, et al. Aminotransferase changes and acute hepatitis in patients with dengue fever: analysis of 1,585 cases. Braz J Infect Dis. 2004;8(2):156-63.

7. Trung DT, Thao LTT, Hien TT, Hung NT, Vinh NN, Hien PTD, et al. Liver involvement associated with dengue infection in adults in Vietnam. Am J Trop Med Hyg. 2010;83(4):774-80.

8. Narasimhan D, Ponnusamy P, Sathish M. Analysis of liver function tests in dengue fever. Int J Adv Med. 2018;5(1):47-9.

9. Assessment of Dengue fever severity through liver function test IntechOpen. https://www.intechopen.com/books/dengue-immunopath ology-and-control-strategies/assessment-of-dengue-fever-severity-throu gh-liver-function-test. Accessed 28 May 2021. 
10. Alberg AJ, Park JW, Hager BW, Brock MV, Diener-West M. The use of 'overall accuracy'to evaluate the validity of screening or diagnostic tests. J Gen Intern Med. 2004;19(5 Pt 1):460-5.

11. Kuo CH, Tai DI, Chang-Chien CS, Lan CK, Chiou SS, Liaw YF. Liver biochemical tests and dengue fever. Am J Trop Med Hyg. 1992;47(3):265-70.

12. Fernando S, Wijewickrama A, Gomes L, Punchihewa CT, Madusanka SDP, Dissanayake $\mathrm{H}$, et al. Patterns and causes of liver involvement in acute dengue infection. BMC Infect Dis. 2016. https://www.ncbi.nlm.nih.gov/ pmc/articles/PMC4938910/. Accessed 31 May 2021.

13. Lee MS, Hwang KP, Chen TC, Lu PL, Chen TP. Clinical characteristics of dengue and dengue hemorrhagic fever in a medical center of southern Taiwan during the 2002 epidemic. J Microbiol Immunol Infect. 2006;39(2):121-9.

14. Kalayanarooj S, Vaughn DW, Nimmannitya S, Green S, Suntayakorn S, Kunentrasai $\mathrm{N}$, et al. Early clinical and laboratory indicators of acute denque illness. J Infect Dis. 1997;176(2):313-21.
15. Green RM, Flamm S. AGA technical review on the evaluation of liver chemistry tests. Gastroenterology. 2002;123(4):1367-84.

16. Textbook of hepatology: from basic science to clinical practice, 3rd edition. Wiley.com. https://www.wiley.com/en-us/Textbook+of+Hepat ology\%3A+From+Basic+Science+to+Clinical+Practice\%2C+3rd+Edit on-p-9781405181518. Accessed 25 Jul 2018

\section{Publisher's Note}

Springer Nature remains neutral with regard to jurisdictional claims in published maps and institutional affiliations.
Ready to submit your research? Choose BMC and benefit from:

- fast, convenient online submission

- thorough peer review by experienced researchers in your field

- rapid publication on acceptance

- support for research data, including large and complex data types

- gold Open Access which fosters wider collaboration and increased citations

- maximum visibility for your research: over 100M website views per year

At BMC, research is always in progress.

Learn more biomedcentral.com/submissions 